\title{
EUSO-Balloon: Observation and Measurement of Tracks from a laser in a Helicopter
}

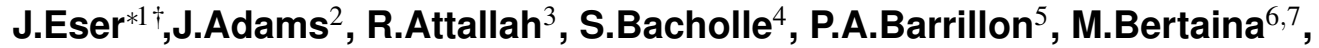 \\ F.Cafagna $^{14}{ }^{\text {, D.Campana }}{ }^{8}$, C.Catalano ${ }^{10}$, M.Christl $^{11}$, S.Dagoret-Campagne ${ }^{12}$, \\ M.Fouka $^{13}$, F.Guarino ${ }^{8,9}$, A.Jung ${ }^{4}$, E.Kuznetsov' ${ }^{2}$, E.Mayotte ${ }^{1}$, C.Moretto ${ }^{12}$, \\ G.Osteria $^{8}$, W.Painter ${ }^{1}$, B.Panico ${ }^{8}$, F.Perfetto ${ }^{8,9}$, G.Prevot ${ }^{4}$, J.A.Rabanal Reina ${ }^{12}$, \\ M.Rodencal $^{2}$, Z.Sahnoun ${ }^{13}$, J.Sawatzki ${ }^{2}$, V.Scotti ${ }^{8,9}$, G.Suino ${ }^{6,7}$, P.vonBallmoos $^{10}$, \\ L.Wiencke ${ }^{1}$ \\ ${ }^{1}$ Colorado School of Mines \\ ${ }^{2}$ University of Alabama,Huntsville \\ ${ }^{3}$ University of Annaba \\ ${ }^{4}$ APC-Paris Diderot university \\ ${ }^{5}$ Universite de Paris-Sud 11 \\ ${ }^{6}$ Universita Torino \\ ${ }^{7}$ INFN, Torino \\ ${ }^{8}$ INFN, Napoli \\ ${ }^{9}$ Universita di Napoli Federico II - Dipartimento di Fisica \\ ${ }^{10}$ IRAP \\ ${ }^{11} N A S A, M S F C$ \\ ${ }^{12}$ LAL/IN2P3/CNRS \\ ${ }^{13}$ Dep. Astronomy, Centre Res. Astronomy, Astrophysics and Geophysics \\ ${ }^{14}$ INFN Bari
}

\section{for the JEM-EUSO Collaboration}

EUSO-Balloon is a prototype detector of the Extreme Universe Space Observatory on the Japanese Experiment Module (JEM-EUSO). EUSO-Balloon was flown successfully as a balloon payload from the Timmins Stratospheric Balloon Launch Facility in Ontario, Canada on 2014 August 24-25 at an altitude of $38 \mathrm{~km}$. To simulate the optical signatures of UV fluorescence photons emitted from cosmic ray air showers generated in the atmosphere, a pulsed UV laser and two UV flashers (LED and Xe) were used. These sources were fired in the instrument field of view for about 2 hours from a helicopter that circled at an altitude of $3 \mathrm{~km}$ under the balloon. UV signals were effectively detected, including 270 laser track events. We describe the helicopter laser system and the geometric reconstruction of the laser events that were generated by this system. We report here on the reconstruction of the laser events starting from the information contained in the observed tracks. We note that this work represents the first observation and measurement of aircraft based laser tracks by an optical fluorescence detector flown at near space altitudes.

The 34th International Cosmic Ray Conference,

30 July- 6 August, 2015

The Hague, The Netherlands 

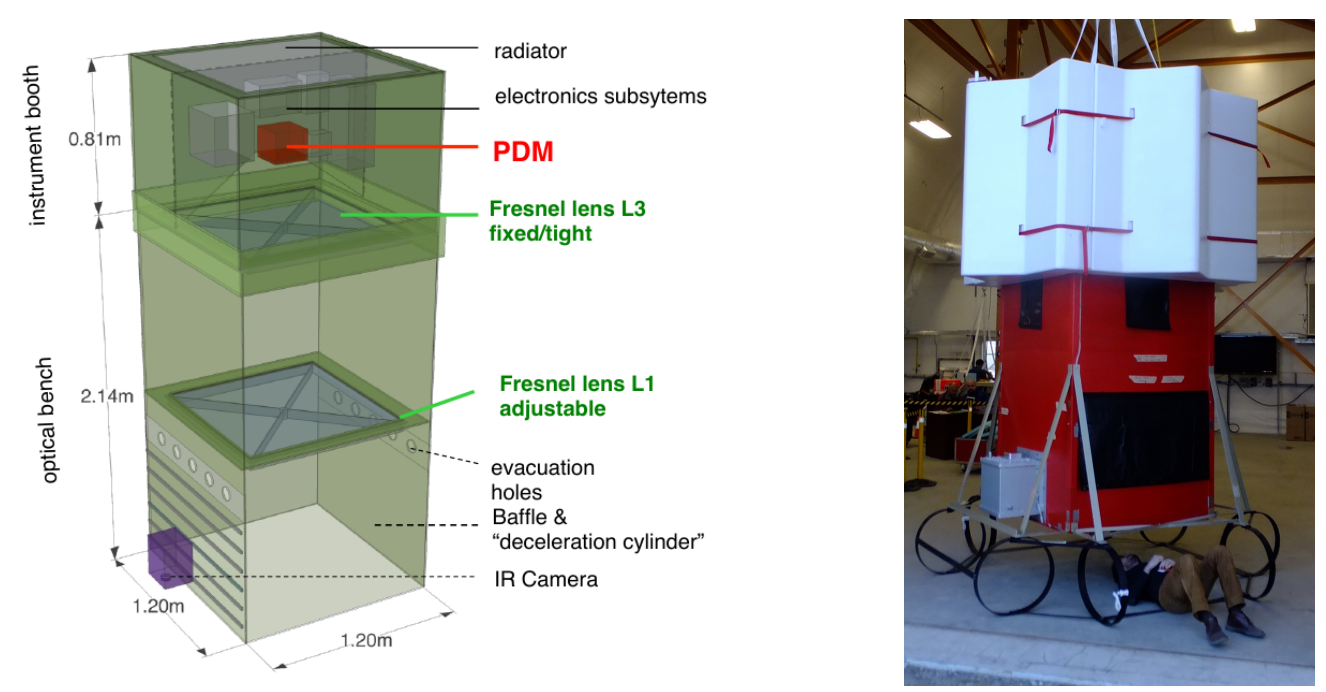

Figure 1: left: drawing of the flown EUSO-Balloon detector [1]; right: the actual detector

\section{EUSO-Balloon Mission}

EUSO-Balloon is a prototype detector of the Extreme Universe Space Observatory on the Japanese Experiment Module (JEM-EUSO). JEM-EUSO is a UV telescope that will observe Extremely Energetic Cosmic Rays (EECR) from the International Space Station (ISS) by measuring the UV light produced by EECR-induced extensive air showers(EAS) in the earth's atmosphere.

To perform a full scale test in a near space environment and to measure the earth UV background from above, the EUSO-Balloon detector was flown as the payload on a stratospheric balloon. This balloon was launched from the Timmins (Canada) stratospheric balloon launch facility during the night of the 24th/25th of August 2014 under the lead of the French space agency (CNES) and with support of the Canadian space agency(CSA ASC).

The EUSO-Balloon instrument(Fig. 1) uses two $1 \mathrm{~m}^{2}$ Fresnel lenses to focus light onto the Photo-Detector Module (PDM) in the focal surface. The PDM consists of 36 Hamamatsu M64 multi-anode photomultipliers tubes (MAPMTs) each containing 64 anodes that are capable of single photon counting. These 2304 pixels detect light between 290 and $430 \mathrm{~nm}$ through a BG3 filter. The field of view $(\mathrm{FoV})$ of a single pixel is $0.23^{\circ}$. The $\mathrm{FoV}$ of the instrument is $11^{\circ} \times 11^{\circ}$. During the flight the detector recorded short "video clips" at a rate of $20 \mathrm{~Hz}$. Each clip consists of $128 \mathrm{con}-$ secutive $2.5 \mu$ s frames of the all 2304 pixels or Gate Time Unit (1GTU). Details about the detector are described in [2]. The payload was also equipped with an IR camera for cloud detection ([3, 4]).

To test the capability of the detector to measure UV light from an EAS, 3 light sources were fired from a helicopter that circled under the balloon. The arrangement is illustrated in Fig. 3. The wavelengths of the 3 sources (UV LED, Xe-Flashlamp, UV laser) were similar to the wavelengths of fluorescence emission of electrons in air (Fig. 2). The nominal laser energy was equivalent to the light emitted by a $100 \mathrm{EeV}$ EAS [6]. The LED and the Xe-flashlamp were mounted in a basket outside of the Bell 212 Helicopter (see Fig. 4). The laser system was fixed to the inside of the

\footnotetext{
* Speaker.

†jeser@mines.edu
} 


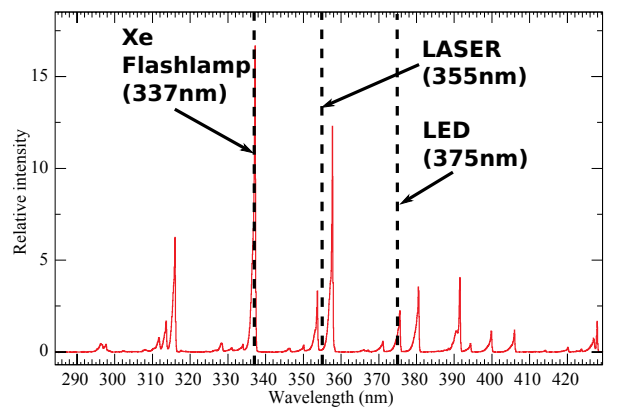

Figure 2: The wavelengths of the LED (375 nm), the laser (355 nm) and filtered Xe flashlamp (337 $\mathrm{nm})$ are indicated on the fluorescence spectrum of electrons in air [5].

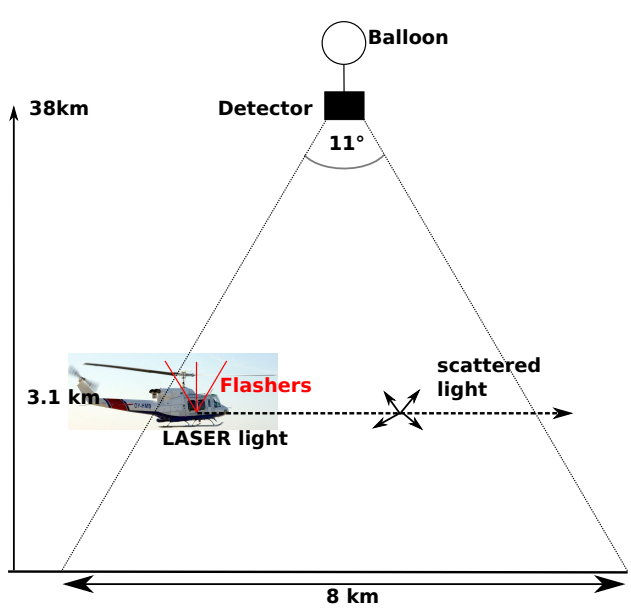

Figure 3: Sketch of the helicopter underflight

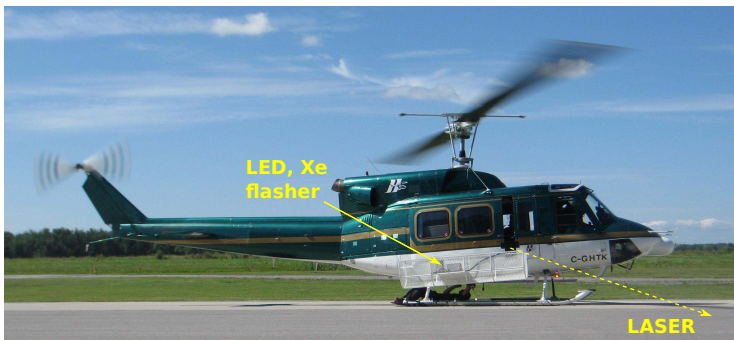

Figure 4: The light sources were mounted on a Bell 212 helicopter. This dual turbine model was used because it satisfied various rules for night flight in Canada.

helicopter. The beam was aimed out a partially open door to fire perpendicular to the front- back axis of the helicopter and horizontally when the helicopter was flying level.

The trajectories of the balloon and the helicopter are shown in Fig. 5 and the timeline of the mission is shown in Fig. 6. The balloon was launched at 01:00 UTC and reached float altitude of approximately $38 \mathrm{~km}$ after a ascent of 2.5 hours. After 5 hours at float altitude the payload was released from the balloon at 8:30 UTC and landed half an hour later in a lake.

The helicopter arrived from Ottawa on the evening of the launch, refueled in Timmins and followed the balloon using a GPS tracking system. At 03:31 UTC the helicopter entered the FoV of the detector and the light sources were turned on. To trigger pixels across the whole camera, the pilots flew circular loops with a radius smaller then $4 \mathrm{~km}$ centered on the latitude/longitude of the balloon. The flight path is plotted in red in Fig. 5. The sources were fired $\sim 150000$ times in 2.28 hours.

\section{The Light Systems}

The laser was a Quantel CFR-Ultra [7] pulsed YAG-laser with frequency tripling to $355 \mathrm{~nm}$. The maximum energy was $18 \mathrm{~mJ}$. The pulse width was $7 \mathrm{~ns}$. The relative energy of every pulse was measured by a "pickoff" energy probe. The beam was sent through a depolarizer so that the scattering out of the beam in the air would be symmetric in the azimuth angle perpendicular to the 


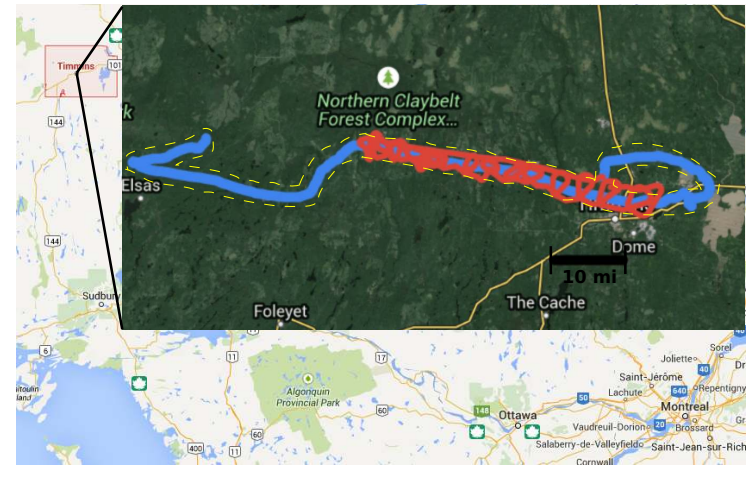

Figure 5: The thick blue line shows the flight path of the balloon. The yellow dashed line is the approximate FoV. The red line (loops) shows the flight pattern of the helicopter while the light sources were firing.(created with google maps)

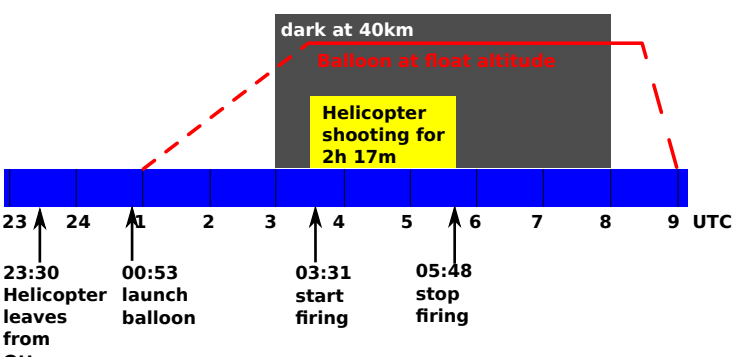
Ottawa

Figure 6: Timeline of the balloon and helicopter mission. The dashed line indicates the balloon.

beam axis. The full optical setup is shown in Fig. 7. The beam characteristics are listed in Table 1. The laser system was calibrated before and after the flight by measuring the ratio between the monitor energy probe and a second energy probe placed temporarily in the beam downstream of all optics. The difference between this calibration factor measured before and after the flight was $1.2 \%$. A description of the flashers and their different operation modes can be found in [8].
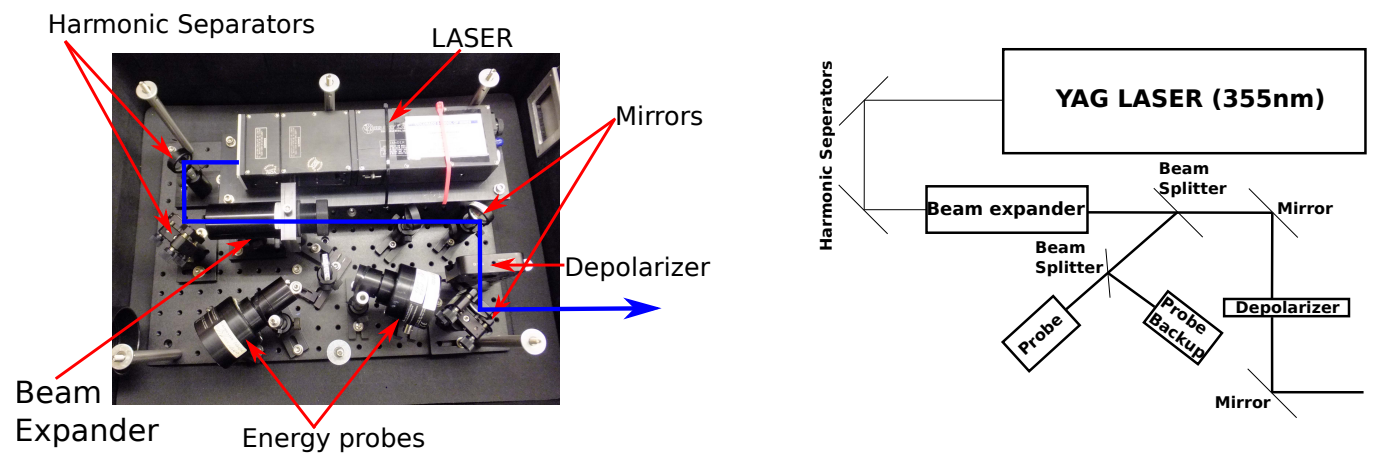

Figure 7: Left: The UV-laser system that was flown in the helicopter. The thick blue line indicates the beam path. Right: Schematic of the UV-laser system

The three sources were triggered in a specific order (1.LED, 2.Laser, 3. Xe-Flasher) by a Single Board Computer equipped with a custom GPS module for a precise timing [9]. The sequence was timed so that light from the three sources could arrive in the same 128 GTU readout window. The laser energy was switched every two minutes between $\sim 15 \mathrm{~mJ}$ and $\sim 10 \mathrm{~mJ}$. A $19 \mathrm{~Hz}$ repetition rate was chosen to obtain a chance overlap at regular intervals between the $20 \mathrm{~Hz}$ readout of the balloon and the laser. This arrangement was implemented to work around a problem with the clock synchronization between the two systems, probably caused by a faulty GPS antenna on the balloon. The average energy of the shots, measured at the laser system, as a function of time can be seen in Fig. 8. The 270 laser shots that were recorded by EUSO-balloon are superimposed. Most of the events were recorded when there were no clouds between the laser and the balloon. 


\begin{tabular}{c|c|c|c} 
wavelength & $355 \mathrm{~nm}$ & divergence & $<0.04^{\circ}$ \\
\hline relative energy calibration & $>2 \%$ & beam halo & $<0.5 \%$ \\
\hline absolute energy calibration & $>4.5 \%$ & spectral purity & $>99.9 \%$ \\
\hline overall stability & $1.2 \%$ & \multirow{2}{*}{ beam pointing direction } & $\begin{array}{c}\text { vertical: } 3^{\circ} \\
\text { horizontal: } 1^{\circ}\end{array}$ \\
\hline depolarization & $<4 \%$ & &
\end{tabular}

Table 1: Laser beam characteristics

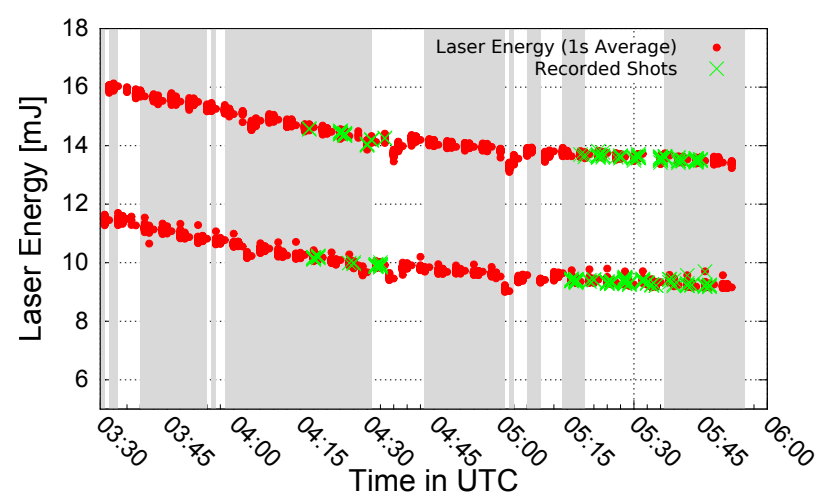

Figure 8: Red dots: Energy of all fired laser shots averaged over 19 shots. Green Xs: Shots recorded by EUSO-balloon. Grey regions indicate the likely presence of clouds.

\section{Geometric Reconstruction}

The EUSO-balloon data for a single event consists of a 128 GTU sequence of the 2304 pixels. From the times and the pointing directions of the pixels with signals above background, we reconstruct the direction of the laser beam relative to the orientation of the detector. The analysis follows two major steps. First the pointing direction of the selected pixels are used to find the Shower Detector Plane (SDP) (Fig. 9). To reconstruct the direction of the event in the SDP, a trial nominal laser direction is estimated. Then the expected time at the detector is calculated for each pixel based on the region of the event axis to which it points (see Eq. 3.1). The difference between the expected and the observed time is compared and the parameters are adjusted to minimize time differences across the camera using the $\chi^{2}$ method. The geometry with the minimum difference is the reconstructed shower axis. The distance of closest approach, $R_{P}$, and the angle from horizontal to $R_{P}, \Psi_{0}$ are the two parameters describing the axis. The arrival time at the $\mathrm{i}^{\text {th }}$ pixel is given by

$$
t_{i, \text { expected }}=T_{0}+\frac{R_{P}}{c} \tan \left(\frac{\pi}{4}+\frac{\psi_{0}-\psi_{i}}{2}\right)
$$

where $\psi_{i}$ is the pointing direction of each participating pixel projected into the SDP. $T_{0}$ is the time when the shower front reaches $R_{P}$. The scattered laser light is assumed to travel at the speed of light (c) and the detector is assumed to point directly down. If the change in angular speed $\frac{d \psi}{d t}$ is small over the observed track length (low curvature in time vs angle), the uncertainties in the 3 parameter fit can be large. 


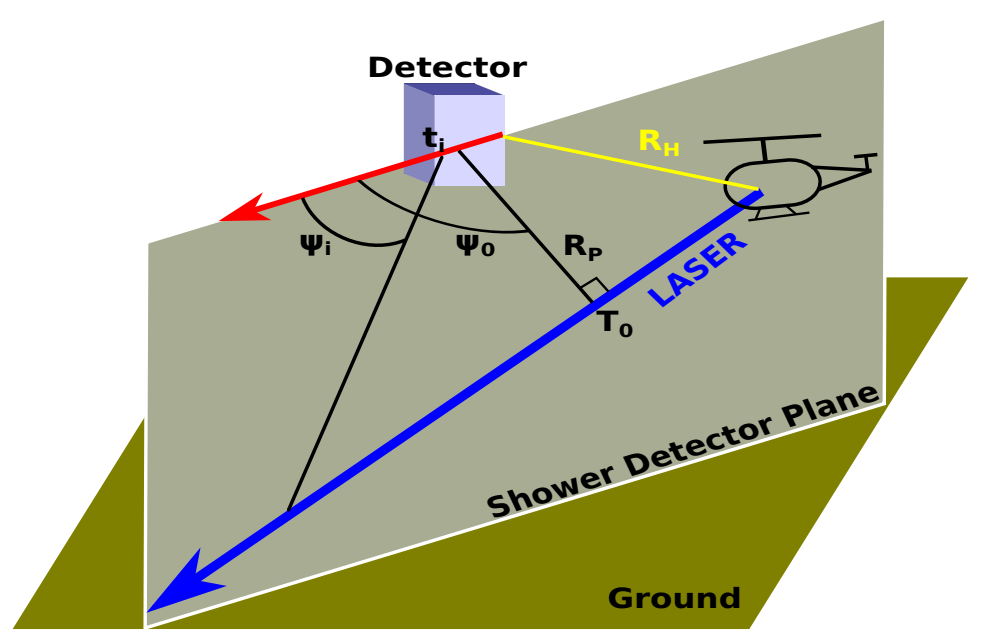

Figure 9: Illustration of the geometrical direction reconstruction of the laser tracks fired from the helicopter with observables of the balloon.

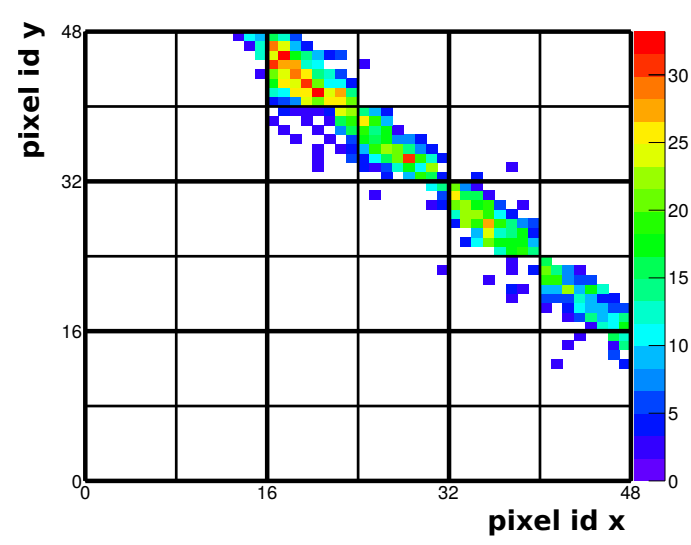

(a) laser track in the PDM. Colour represents the relative charge

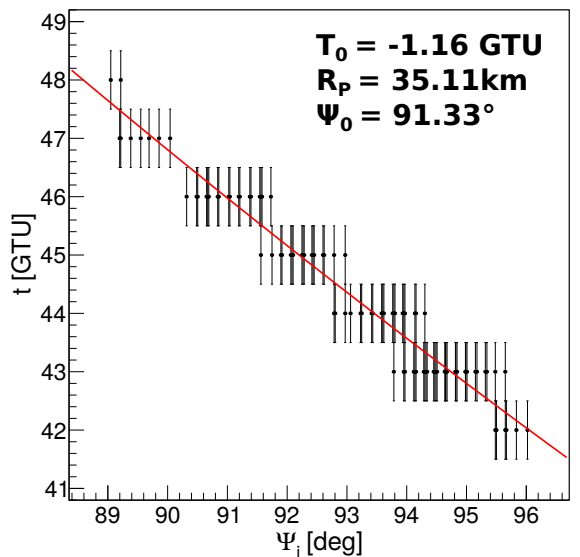

(b) Timing profile of a laser event with constant 0.5 GTU timing error.

Figure 10: Example laser track at 05:29:45 UTC with corresponding time profile and fit.

A constraint fit was performed using the known position of the helicopter. This reduces the number of parameters in Eq.(3.1) from 3 to $1\left(\Psi_{0}\right)$ [10]. The time expected at the detector is then given by

$$
t_{i, \text { expected }}=t_{H}-\frac{R_{H}}{c}\left(1-\sin \left(\psi_{H}-\psi_{0}\right)\right)+\frac{R_{H} \cos \left(\psi_{H}-\psi_{0}\right)}{c} \tan \left(\frac{\pi}{4}+\frac{\psi_{0}-\psi_{i}}{2}\right)
$$

where $R_{H}$ is the distance between the detector and the helicopter. $t_{H}$ is the laser firing time plus propagation time and $\psi_{H}$ is the angle between the detector and the helicopter. $\psi_{H}$ and $t_{H}$ are determined by the first occurrence in the camera.

The data was analysed using the JEM-EUSO Offline framework [11]. An example laser track with the corresponding timing fit is shown in Fig. 10. 


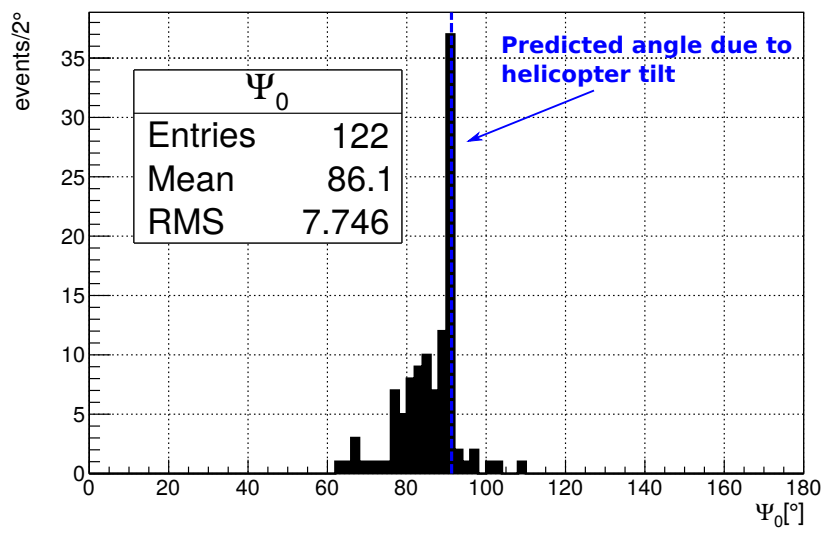

Figure 11: Zenith angle reconstruction of the helicopter laser shots with the 1 parameter fit method

\section{Reconstruction Results}

The reconstruction of $\Psi_{0}$ for the full data set using the constraint fit (Eq. 3.2) is shown in Fig. 11. The distribution has a mean reconstructed zenith angle of $86.1^{\circ}$ and a root mean square (RMS) of $7.75^{\circ}$ (only noise cuts applied). This resolution does not represent the final resolution of the instrument and will likely be improved by applying calibration data. The time resolution of $2.5 \mu$ s presents another issue as this bin size is too large to achieve an angular resolution within a few degrees at the short distance between the helicopter and the balloon. This is not an issue for JEM-EUSO, because the detector-shower separation is around 10 times bigger. The most common values in this distribution are between $90^{\circ}$ and $92^{\circ}$. This means the majority of the laser tracks were reconstructed to point slightly towards the ground as expected considering the helicopter flight pattern. To fly in a circular pattern, the helicopter was tilted slightly towards the ground by approximately $1-2^{\circ}$ and the laser fired out of the "lower" side of the helicopter. The estimation of this angle can be found using the measured velocity of the helicopter and the assumption of a circular flight pattern $\left(\theta=\arcsin \left(\frac{v^{2}}{R \cdot g}\right)\right)$. The difference between the mean and the mode of this distribution is not yet understood and needs more investigation. Another work in progress is the described fit with no constrains ( 3 parameter). Due to the flat timing profile of the laser tracks in the camera, a worse resolution is expected with the 3 parameter fit compared to the 1 parameter fit. This prediction remains to be quantified, especially after applying IR camera and calibration data.

\section{Conclusion}

EUSO-balloon made the first measurements of tracks from above looking down. The recording of the 270 events was a success for the balloon mission. Cloud attenuation was expected for EUSOballoon. However, this is not a significant issue for JEM-EUSO, as often most of the shower will develop above the clouds. Constraining the detected track to start at the helicopter position at the time of the first occurrence of the laser light in the camera minus the light propagation time, gives a mean zenith angle of $86.1^{\circ}$ and an RMS of $7.75^{\circ}$. The shift to a slightly downward pointing direction, regarding the mode $\left(90-92^{\circ}\right)$, agrees with the magnitude and direction of the tilt of the 
helicopter as it circled while the laser was firing. These results are better than expected regarding the fact the EUSO-balloon was the first prototype detector for JEM-EUSO with the main purpose to test the hardware components in a near space environment.

Acknowledgment: The authors acknowledge strong support from the French Space Agency CNES. This work was partially supported by Basic Science Interdisciplinary Research Projects of RIKEN and JSPS KAKENHI Grant (22340063, 23340081, and 24244042), by the Italian Ministry of Foreign Affairs, General Direction for the Cultural Promotion and Cooperation, by the 'Helmholtz Alliance for Astroparticle Physics HAP' funded by the Initiative and Networking Fund of the Helmholtz Association, Germany, and by Slovak Academy of Sciences MVTS JEM-EUSO as well as VEGA grant agency project 2/0076/13. Russia is supported by the Russian Foundation for Basic Research Grant No 13-02-12175-ofi-m. The US is supported by the NASA grants NNX13AH55G, NNX13AH53G. The Spanish Consortium involved in the JEM-EUSO Space Mission is funded by MICINN \& MINECO under the Space Program projects: AYA2009-06037-E/AYA, AYA-ESP2010-19082, AYA-ESP2011-29489-C03, AYA-ESP2012-39115C03, AYA-ESP2013-47816-C4, MINECO/FEDER-UNAH13-4E-2741, CSD2009-00064 (Consolider MULTIDARK) and by Comunidad de Madrid (CAM) under projects S2009/ESP-1496 \& S2013/ICE-2822.

\section{References}

[1] P. von Ballmoos et al. (JEM-EUSO Collaboration), EUSO-balloon : A pathfinder for observing uhecr's from space in Proc. 33th ICRC, (Rio de Janeiro),(2013).

[2] P. von Ballmoos et al. (JEM-EUSO Collaboration), General overview of EUSO-balloon mission in Proc. 34th ICRC, (The Hague), (2015).

[3] M. Frias et al. (JEM-EUSO Collaboration), The infrared camera onboard the EUSO-balloon (CNES) flight on August 24, 2015 in Proc. 34th ICRC, (The Hague), (2015).

[4] J. Fernandez et al. (JEM-EUSO Collaboration), Performances of the infrared camera onboard the EUSO-balloon (CNES) flight on August 24, 2014 in Proc. 34th ICRC, (The Hague), (2015).

[5] The AIRFLY Collaboration, M. Ave et al. Measurement of the pressure dependence of air fluorescence emission induced by electrons, Astropart.Phys. 28:41(2007).

[6] The Pierre Auger Collaboration, P.Abreu et al., Techniques for measuring aerosol attenuation using the Central Laser Facility at the Pierre Auger Observatory, JINST 8, p04009 (2013),[arXiv:13035576v].

[7] http://www. quantel-laser.com.

[8] J. Adams et al. (JEM-EUSO Collaboration), The calibration of EUSO-balloon using airborne light sources mounted to a helicopter in Proc. 34th ICRC, (The Hague), (2015).

[9] S.B. Thomas J.D. Smith, J. R. Thomas and L. R. Wiencke, A programmable hardware module for precise absolute time event generation and capture in Proc. 30th ICRC, (Mexico) 5997 (2008) (US patent 7,975,160).

[10] W. Painter, Estimating the angular resolution of the Extreme Universe Space Observatory (EUSO) using a modifies version of the Pierre Auger Observatory fluorescence detector reconstruction framework, Master's thesis, Colorado School of Mines, 2015.

[11] T. Paul et al. (JEM-EUSO Collaboration), A new design for simulation and reconstruction software for the JEM-EUSO mission in Proc. 34th ICRC, (The Hague), (2015). 